REVIEW ARTICLE

C.S. Bonnet
É. Tóth

\section{Smart MR Imaging Agents Relevant to Potential Neurologic Applications}

SUMMARY: Molecular imaging is aimed at the noninvasive visualization of the expression and function of bioactive molecules that often represent specific molecular signatures in disease processes. Any molecular imaging procedure requires an imaging probe that is specific to a given molecular event, which puts an important emphasis on chemistry development. In MR imaging, the past years have witnessed significant advances in the design of molecular agents, though most of these efforts have not yet progressed to in vivo studies. In this review, we present some examples relevant to potential neurobiologic applications. Our aim was to show what chemistry can bring to the area of molecular MR imaging with a focus on the 2 main classes of imaging probes: Gd $^{3+}$-based and PARACEST agents. We will discuss responsive probes for the detection of metal ions such as $\mathrm{Ca}, \mathrm{Zn}, \mathrm{Fe}$, and $\mathrm{Cu}$, $\mathrm{pH}$, enzymatic activity, and oxygenation state.
$\mathbf{M}_{\mathrm{o}}^{\mathrm{o}}$ olecular imaging is an emerging science area aimed at noninvasive visualization of the expression and function of bioactive molecules that often represent specific molecular signatures in disease processes. It seeks the biochemical or physiologic abnormalities underlying the disease, rather than the structural consequences of these abnormalities. Potential clinical applications of molecular imaging have been widely recognized, though the limited sensitivity and specificity of current molecular imaging approaches are often a major roadblock for clinical applications. Besides clinical settings, drug development can also largely benefit from the integration of molecular imaging. Molecular imaging not only allows rationalization of the parameters related to disease processes but it can also replace the common invasive research techniques (such as histology, which requires sacrificing animals for each time point in the experiment) by time-effective repeatable real-time visualization of biologically relevant processes. Currently, progress in the molecular imaging area is simultaneously moving at 3 levels: 1) improving the imaging hardware for use in preclinical and clinical settings, 2) identifying and validating new biologically relevant imaging targets, and 3) developing innovative imaging probes. Unlike anatomic imaging, molecular imaging always requires a molecular im-

From Centre National de la Recherche Scientifique (CNRS), Centre de Biophysique Moléculaire, Orléans, France.

This work was supported by the National Institute of Cancer, La Ligue contre le Cancer and Agence Nationale de la Recherche, France; as well as the European Cooperation in Science and Technology Action D38 "Metal-Based Systems for Molecular Imaging Applications."

Please address correspondence to: Éva Tóth, PhD, rue Charles Sadron, 45071 Orléans, France; email: eva.jakabtoth@cnrs-orleans.fr

Indicates open access to non-subscribers at www.ajnr.org

DOI 10.3174/ajnr.A1753 aging probe, and this places chemistry development in a central position.

Among all molecular imaging technologies, MR imaging is particularly attractive. Its exceptional resolution allows acquiring anatomic images in conjunction with mapping the activity of biomarkers. Since the introduction of MR imaging into clinical practice, contrast-enhancing agents have been widely used to improve the relatively low sensitivity of the technique. Conventional MR imaging contrast agents are paramagnetic substances that enhance the water proton relaxation rates. Unlike optical or radiodiagnostic probes, the MR imaging agents do not directly generate a signal intensity but are detected via their effect on the water proton relaxation. Given the high paramagnetism of $\mathrm{Gd}^{3+}$ (it has 7 unpaired electrons), its poly(amino carboxylate) complexes are mostly used today in clinical practice. $\mathrm{Gd}^{3+}$ complexes have a more important effect on the longitudinal relaxation times (T1 agents) than on the transverse relaxation times (T2 agents). In the $\mathrm{Gd}^{3+}$ complexes, the poly(amino carboxylate) ligand, such as DTPA or DOTA (Fig 1), wraps around the $\mathrm{Gd}^{3+}$ ion to prevent its toxicity; however, it leaves enough room for 1 water molecule directly linked to the paramagnetic metal ion. The exchange of this water molecule with the surrounding water is extremely important because it will transmit the paramagnetic effect of the Gd to the bulk water to make the molecule detectable as a decreased proton relaxation time (Fig 2).

The currently used clinical agents are nonspecific. While they help delineate the structural anatomic consequences of diseases, they are not capable of indicating the biochemical or physiologic abnormalities underlying them. The efficacy of the agent to accelerate water proton relaxation, defined as relaxivity, has to be selectively influenced by the particular parameter/biomarker that we want to detect, to create a "smart" 


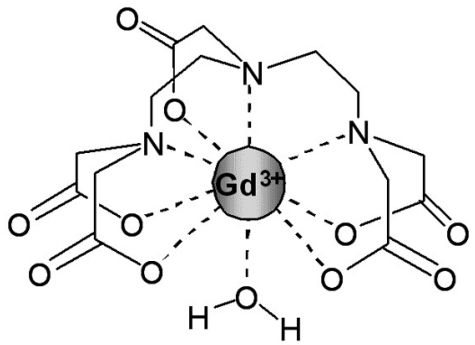

$\operatorname{GdDTPA}\left(\mathrm{H}_{2} \mathrm{O}\right)^{2-}$

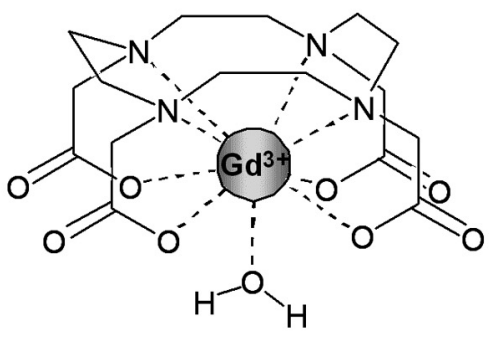

$\operatorname{GdDOTA}\left(\mathrm{H}_{2} \mathrm{O}\right)^{-}$

Fig 1. Gd-DOTA (Dotarem; Guerbet, Aulnay-sous-Bois, France) and Gd-DTPA (Magnevist; Schering, Berlin, Germany). Two examples of clinically used Gd ${ }^{3+}$ polylamino carboxylate) complexes. All other approved $\mathrm{Gd}^{3+}$-based contrast agents are derivatives of Gd-DOTA or Gd-DTPA.

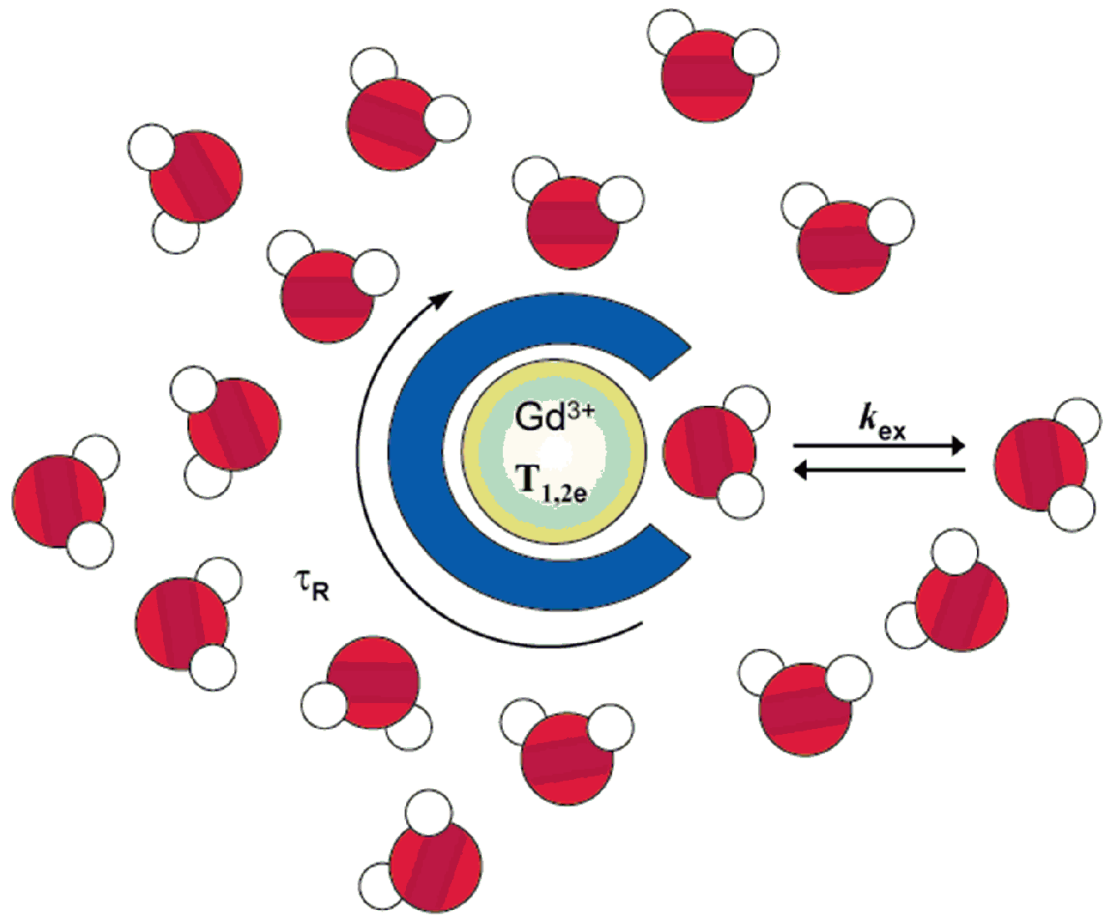

Fig 2. In $\mathrm{Gd}^{3+}$-based contrast agents, the paramagnetic $\mathrm{Gd}^{3+}$ ion is coordinated by an organic ligand. The water molecules directly linked to the metal exchange with bulk water, and this exchange transmits the paramagnetic effect of $\mathrm{Gd}^{3+}$ to the surrounding environment. The efficacy of the agent is mainly related to the number of water molecules bound to $\mathrm{Gd}^{3+}$ (the hydration number), $q$ (the exchange rate of the coordinated water molecule[s] with the surrounding water), $k_{\mathrm{ex}}$, and the rotational correction time $\tau_{\mathrm{R}}$.

(activatable or responsive) contrast agent. The relaxation behavior of paramagnetic complexes and the underlying mechanisms have been extensively discussed elsewhere. ${ }^{1-3}$ Here we only summarize the various modulation pathways for the relaxivity of $\mathrm{Gd}^{3+}$-based agents. The relaxivity (paramagnetic relaxation enhancement of water proton relaxation per millimolar concentration of $\mathrm{Gd}^{3+}$ ) is inherently related to the microscopic properties of the $\mathrm{Gd}^{3+}$ complex. The most important microscopic factors are the following: 1) the number of water molecules directly coordinated to the metal ion (hydration number), 2) the exchange rate of these water molecules with the surrounding water, and 3 ) the motion dynamics of the molecule (how fast the complex tumbles), usually characterized by the reorientational correlation time of the agent (Fig 2). In principle, any of these parameters could be modulated; however, most of the smart contrast agents function on the basis of changes in the hydration number or in the reorientational correlation time of the molecule because they are the easiest to predict and modulate by chemical changes. The relaxivity is linearly proportional to the hydration number. The reorientational correlation time of the agent is determined by the size and the flexibility of the molecule. Generally speaking, slower rotation (larger size and reduced flexibility) translates to higher relaxivity of the $\mathrm{Gd}^{3+}$ complex. However, this effect is strongly dependent on the magnetic field, and vanishes at fields above $\sim 4 \mathrm{~T}$.

Recently, a new class of potential paramagnetic MR imaging contrast agents, based on CEST, has been explored as a promising alternative to classic relaxivity agents. ${ }^{4-8}$ In particular, they have generated a great enthusiasm as activatable probes. PARACEST agents are complexes of lanthanide ions other than gadolinium, which possess paramagnetically shifted exchangeable protons. Those protons $(-\mathrm{NH}$ of amides, amines, or $-\mathrm{OH}$ of water or alcohols) are in slow exchange with bulk water protons (Fig 3). They can be selectively saturated by radio-frequency pulses applied at the ap- 


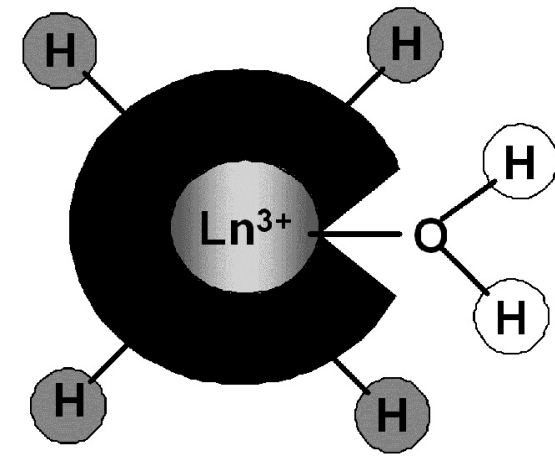

Fig 3. Schematic representation of a PARACEST agent with exchangeable protons on the ligand (amine, amide, alcohol...) (gray) or on the coordinated water (white).

propriate frequency, and due to the chemical exchange between these and the water protons, their saturation will result in a decrease of the water proton signal intensity, which becomes observable on an MR image. PARACEST sensors are based on modulation of the exchange rate or the resonance frequency of those mobile protons on the agent. The concept of chemical exchange saturation transfer can also be applied to nonparamagnetic endogenous molecules; however, the signals of their protons are located close to the bulk water signal intensity, which hampers their selective saturation. PARACEST probes are ideally suited for molecular imaging because contrary to $\mathrm{Gd}^{3+}$-based MR imaging agents, the contrast can be switched on and off at will by simply adjusting the pulse sequence parameters. Another advantage of PARACEST with respect to relaxivity agents is the possibility of applying ratiometric approaches that eliminate the problem of unknown local concentration of the agent and allow quantitative determination. Such ratiometric approaches involve the use of a cocktail of 2 isostructural complexes: one as a reporter of a physiologic parameter and the other as a simple concentration marker, whereas both have an identical biodistribution.

In recent years, significant progress has been made in the design of novel molecular MR imaging probes, though very few of them have entered in vivo studies. With respect to neuroimaging, many pathologies are associated with an altered permeability or disruption of the $\mathrm{BBB}$; however, for a contrast agent, crossing the intact BBB still remains a challenge. In this review, we present some examples of recent development of molecular MR imaging probes relevant to neurobiologic applications. It is not intended to be an exhaustive review but rather an illustration of what chemistry can bring to molecular MR imaging. Other reviews related to molecular neuroimaging, not restricted to MR imaging, have been recently published. ${ }^{9-11}$ Here we will discuss paramagnetic lanthanide complexes involving $\mathrm{Gd}^{3+}$ agents and PARACEST probes and will focus on responsive agents designed to detect metal ions, such as $\mathrm{Ca}^{2+}, \mathrm{Zn}^{2+}, \mathrm{Cu}^{2+}$, and $\mathrm{Fe}^{2+/ 3+}, \mathrm{pH}$, enzymatic activity, and oxygenation state. We have included the above metal ions because disruption of their homeostasis is associated with various neurodegenerative diseases. Changes in the extracellular $\mathrm{pH}$ and enzymatic activities can also be the signature of specific pathology processes. The in vivo noninvasive detection of abnormalities in the concentration of these metal ions, in $\mathrm{pH}$, or in enzymatic activities might serve in the future as an important diagnostic tool of the underlying diseases. Informa- tion on the redox status, which is an important factor governing tumor aggressiveness, can help choose the adapted tumor treatment. We should note that many of these abnormalities are observable in the extracellular media, which largely facilitates the chemical design of the imaging probes (no need for intracellular delivery).

\section{Imaging Probes Responsive to Metal Ions}

\section{Ca Sensing}

Understanding how the brain works involves both a comprehension of the physiologic functioning of its individual elements (neurons and glia cells) and a description of their functional architecture and the underlying networks. The activity of networks can be studied with in vivo neuroimaging, in particular with BOLD functional MR imaging. The BOLD technique has, however, physiologic limitations linked to the vascular origin of the signal intensity. ${ }^{12}$ The temporal resolution is in the range of several seconds, and the relationship between the BOLD signal intensity and the neural activity is still not fully understood. One step further could be the use of bioactivated contrast agents as functional markers for processes directly linked to neural signaling. For tracking the neural activity, several specific markers might be envisaged, being responsive to the concentration of certain ions, neurotransmitters, or transmembrane potentials. Very important work in this direction has been performed during the past years by imaging fluorescence signals reporting intracellular $\mathrm{Ca}^{2+}$ fluctuations. ${ }^{13-15}$ However, the intrinsic depth limitations associated with optical imaging restrict these applications to superficial regions.

The extracellular concentrations of $\mathrm{Ca}^{2+}$ play an important role in both physiologic and pathologic processes. $\mathrm{Ca}^{2+}$ ions are not only crucial in several steps in neuronal signaling during normal brain activity but are also associated with a variety of pathologies such as ischemia, hypoglycemia, and seizures. ${ }^{16}$ The involvement of $\mathrm{Ca}^{2+}$ has been implicated in neurometabolic disorders such as in Parkinson disease, ${ }^{17}$ Huntington disease, ${ }^{18}$ amyotropic lateral sclerosis, ${ }^{19}$ schizophrenia, ${ }^{20,21}$ muscular dystrophy, ${ }^{22}$ etc, suggesting a broad spectral role of $\mathrm{Ca}$ in neuropsychiatric disorders. While $\mathrm{Ca}^{2+}$ has been an important target for MR imaging probes, so far, to our knowledge, no agents responsive to neurotransmitters or transmembrane potentials have been reported.

Although strictly speaking, it is not a $\mathrm{Ca}^{2+}$-responsive probe, $\mathrm{Mn}^{2+}$ has been widely used to detect neural activity and to obtain information between interconnected groups of neu-

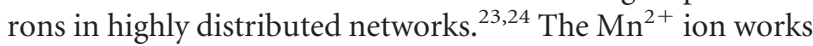
as a paramagnetic mimic of $\mathrm{Ca}^{2+}$; therefore, it accumulates in neurons in an activity-dependent manner. However, Mn-enhanced MR imaging has several drawbacks. The MR imaging signal intensity is proportional to the $\mathrm{Mn}^{2+}$ concentration in tissue; thus, significant amounts of $\mathrm{Mn}^{2+}$ are required to produce detectable contrast. Elevated $\mathrm{Mn}^{2+}$ concentrations can perturb the circuits under study and lead to toxic effects. On the other hand, the very slow uptake of $\mathrm{Mn}^{2+}$ (from hours to days) prevents real-time Ca imaging.

Attempts toward $\mathrm{Ca}^{2+}$-sensitive MR imaging probes involve mainly 2 different approaches: 1) Gd-complexes with a $\mathrm{T} 1$ response on interaction with $\mathrm{Ca}^{2+}$ ions, and 2) a T2 agent 


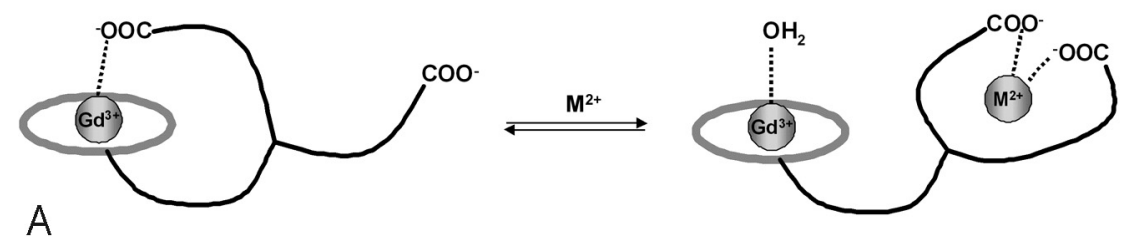

B
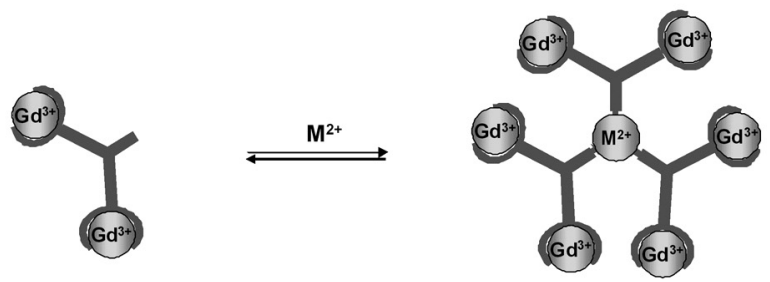

Fig 4. Illustration of 2 different strategies to modulate relaxivity of $\mathrm{Gd}^{3+}$ complexes by cation recognition. $A$ and $B$, Change in the $q(A)$ and change in the size, hence the $\tau_{\mathrm{R}}(B)$. The binding site for the sensed metal ion has to be highly selective with respect to other endogenous cations.

based on the $\mathrm{Ca}^{2+}$-related aggregation of superparamagnetic iron nanoparticles and calmodulin. ${ }^{25}$ The main limitation of this second approach is the relatively long time course of the Ca-dependent aggregation (a few seconds), preventing sensing of fast $\mathrm{Ca}$-concentration changes.

It is much easier to achieve a fast magnetic response by intramolecular interactions. Therefore, $\mathrm{T} 1$ agents $\left(\mathrm{Gd}^{3+}\right.$ complexes) are more favorable with respect to the time course of the Ca-dependent MR imaging response. ${ }^{26}$ The design of all $\mathrm{Gd}^{3+}$-based $\mathrm{Ca}^{2+}$ ion-sensitive probes reported so far involved changes in the coordination sphere of the $\mathrm{Gd}^{3+}$ ion following coordination of $\mathrm{Ca}^{2+}$. The probes integrate 2 coordinating units that selectively chelate $\mathrm{Gd}^{3+}$ and $\mathrm{Ca}^{2+}$ ions. In the absence of the sensed $\mathrm{Ca}^{2+}$ ion, 1 (or more) of the donor groups of the Ca chelating center is weakly coordinated to the $\mathrm{Gd}^{3+}$ ion. On interaction with $\mathrm{Ca}^{2+}$, this donor group will switch from $\mathrm{Gd}^{3+}$ to participate in the $\mathrm{Ca}^{2+}$ coordination, thus liberating 1 coordination position on the $\mathrm{Gd}^{3+}$ ion. The coordination position becoming free around the $\mathrm{Gd}^{3+}$ is immediately occupied by a water molecule (Fig 4A), and the increase of the hydration number results in a relaxivity increase. This approach was used in the design of a series of compounds with 2 macrocyclic chelators connected by a spacer containing a Ca-selective chelating unit. The first example was reported by $\mathrm{Li}$ et al. ${ }^{27}$ Their sensor responded to $\mathrm{Ca}^{2+}$ in the micromolar range, corresponding to intracellular concentrations. However, not only the intracellular delivery of the agent is problematic, but given the low relaxivity of the agent, it is not MR imaging-detectable at micromolar concentrations, which would be needed to sense $\mathrm{Ca}^{2+}$ in the micromolar range.

Targeting the extracellular $\mathrm{Ca}^{2+}$ seems more reasonable for MR imaging applications. The concentration of free extracellular $\mathrm{Ca}^{2+}$ is in the millimolar range and drops up to $30 \%-$ $35 \%$ from the resting state during intense stimulation (typically from 1.2 to $0.8 \mathrm{mmol} / \mathrm{L}) .^{9}$ Sensing $\mathrm{Ca}^{2+}$ changes at millimolar concentrations is more compatible with the relatively high concentrations of the magnetic imaging probe needed to detect contrast changes. Furthermore, targeting extracellular $\mathrm{Ca}^{2+}$ also simplifies the chemical design, because additional requirements for cell internalization can be neglected. A series of $\mathrm{Gd}^{3+}$ complexes have been reported for extracellular $\mathrm{Ca}^{2+}$ sensing ${ }^{28-31}$; the best compounds show $\sim 10 \%$ relaxivity change under biologically relevant conditions (in brain extracellular fluid, for a $\mathrm{Ca}^{2+}$-concentration change between 0.8 and $1.2 \mathrm{mmol} / \mathrm{L}$ ) with a high selectivity for $\mathrm{Ca}^{2+}$, which could already be detectable on MR images.

\section{Zn Sensing}

$\mathrm{Zn}$ is the second most abundant transition metal in the body, and its highest concentrations occur in the brain. Neuronal zinc is partitioned into 2 main classes: a static pool of $\mathrm{Zn}$ that is tightly bound to various metalloproteins and a labile pool of $\mathrm{Zn}$ that is mobile. More than $90 \%$ of the $\mathrm{Zn}^{2+}$ found in the brain and the body is classified as static, playing structural roles in transcription factors and related proteins and structural and catalytic roles in enzymes. The primary source of labile $\mathrm{Zn}^{2+}$ in the brain is from $\mathrm{Zn}^{2+}$ release into the synapse during transmission and can reach concentrations of $\leq 200$ $300 \mu \mathrm{mol} / \mathrm{L}$. Exposure to uncontrolled concentrations of a labile pool of $\mathrm{Zn}^{2+}$ can lead to excitotoxic neuronal death, particularly during epileptic seizures, head trauma, cerebral ischemia and reperfusion, and related situations of overintense neural activity. ${ }^{11}$ In addition to these, long-term disturbances in $\mathrm{Zn}^{2+}$ homeostasis have also been implicated in aging and age-related neurodegenerative diseases such as Alzheimer disease. Indeed, the $\mathrm{Zn}^{2+}$ ion can reach $\sim 1 \mathrm{mmol} / \mathrm{L}$ concentration in amyloid plaques and is implicated in their formation by aggregation of the amyloid- $\beta$ peptide. ${ }^{32}$

As for $\mathrm{Ca}^{2+}$, the main strategy to detect $\mathrm{Zn}^{2+}$ ions is based on a change in the number of water molecules directly bound to the $\mathrm{Gd}^{3+}$ center of a bipartite responsive agent (Fig $4 A$ ). Hanaoka et al studied a system based on the association of a DTPA core and TPEN as a specific $\mathrm{Zn}^{2+}$ chelator. The main drawback of this system is that it can form 1:1 and 2:1 Zn:Gdcomplex adducts leading respectively to a decrease and a further increase in relaxivity on $\mathrm{Zn}^{2+}$ complexation. This situation is problematic because one can reach the same relaxivity value for 2 different amounts of $\mathrm{Zn}^{2+} .{ }^{33}$ The system has been further modified to replace 1 pyridine unit of each TPEN by a carboxylate function, to solve this problem. ${ }^{34}$ Similarly, Major et al studied a series of macrocyclic complexes associated with various $\mathrm{Zn}^{2+}$ chelators such as TPEN or a diaminoacetate arm. They showed that at least 1 acetate arm involved in $\mathrm{Zn}^{2+}$ 
chelation was necessary to observe an increase in relaxivity of about $100 \%$ on $\mathrm{Zn}^{2+}$ coordination. ${ }^{35}$ These probes have not been tested in vivo, but the in vitro MR images showed that the diaminoacetate system is sensitive enough to detect physiologically relevant concentrations of $\mathrm{Zn}^{2+}(\sim 100 \mu \mathrm{mol} / \mathrm{L}) .{ }^{36}$ One highly sensitive PARACEST agent responsive to $\mathrm{Zn}^{2+}$ has been reported by Trokowski et al. ${ }^{37}$ The $\mathrm{Zn}^{2+}$ chelator consists of 2 TPEN units linked to a cyclen core for $\mathrm{Eu}^{3+}$ complexation. On $\mathrm{Zn}^{2+}$ binding, the PARACEST behavior undergoes dramatic changes. ${ }^{37}$ It was explained by an acceleration of the proton exchange, though the underlying mechanism has not been identified. The system is selective for $\mathrm{Zn}^{2+}$ over $\mathrm{Mg}^{2+}$ or $\mathrm{Ca}^{2+}$ ions and is estimated to be able to detect changes in concentration of labile $\mathrm{Zn}$ ranging from $5 \mathrm{nmol} / \mathrm{L}$ to $0.12 \mu \mathrm{mol} / \mathrm{L} .{ }^{37}$

\section{Fe Sensing}

Fe is the most abundant transition metal in the body and in the brain. Indeed, this organ has the highest rate of oxidative metabolism in the body and uses Fe as a key component of enzymes involved in oxygen transport and metabolism. Biologic iron is most commonly found in the +2 (ferrous) and +3 (ferric) oxidation states. $\mathrm{Fe}^{2+/ 3+}$ concentrations in the extracellular environment are in the low micromolar range in the CSF and are typically between 20 and $30 \mu \mathrm{mol} / \mathrm{L}$ in the blood serum of a healthy adult. Intracellular Fe concentrations in neurons range from 0.5 to $1 \mathrm{mmol} / \mathrm{L}$. The major part of Fe in the brain is tightly held by the storage protein ferritin. Exchangeable $\mathrm{Fe}$ in the brain has been proposed to exist in the intracellular environment, in a store termed the "labile iron pool," containing Fe bound by small anions $\left(\mathrm{PO}_{4}{ }^{3-}, \mathrm{CO}_{3}{ }^{2-}\right.$, citrate), polypeptides, and surface components of membrane. However, the existence of this pool is still under active debate. ${ }^{11}$ Fe undoubtedly accumulates in the brain with age and is associated with neurodegenerative diseases, including Alzheimer and Parkinson diseases.

Because the coordination chemistry of $\mathrm{Fe}^{2+}$ and $\mathrm{Fe}^{3+}$ is very different, sensors have to be developed specifically for each oxidation state. $\mathrm{Fe}^{2+}$ is well known for forming stable complexes with phenanthroline, bpy, and tpy ligands in 1:3 and 1:2 metal:ligand ratios. When attached to $\mathrm{Gd}^{3+}$ chelates, such ligands can promote the formation of supramolecular assemblies in the presence of $\mathrm{Fe}^{2+}$. Despite the fact that these systems were mainly studied as contrast agents for imaging at high magnetic fields, they could potentially be used for sensing $\mathrm{Fe}^{2+}$ ions. Indeed, these assemblies formed around the $\mathrm{Fe}^{2+}$ core have a larger size than the monomer $\mathrm{Gd}^{3+}$ complex alone (Fig $4 B$ ), which results in a reduced rotational mobility leading to a relaxivity enhancement. In the case of the phenanthroline derivatives, an increase of $90 \%$ in the relaxivity is observed on the formation of the heterobimetallic tris complex. ${ }^{38}$ The same phenomenon was observed for bpy and tpy derivatives. The former shows an increase of about $200 \%$ at $40 \mathrm{MHz}$ on the formation of the heterobimetallic complex (metallostar), giving rise to an exceptionally high relaxivity confined to a small molecular space. ${ }^{39,40}$ This high in vitro relaxivity is retained under in vivo conditions in mice. The pharmacokinetics of this metallostar, injected intravenously, were found to be similar to that of Gd-DOTA, involving fast renal clearance and a leakage to the extracellular space in the muscle tissue. ${ }^{41} \mathrm{Re}-$ cently, another high-molecular-weight tetrametallic su- pramolecular complex [(Gd-DTPA-phenantroline $\left.)_{3} \mathrm{Fe}\right]$ has been obtained on self-assembly around $1 \mathrm{Fe}^{2+}$ ion. The slower elimination of the compound from rats as compared with $\mathrm{Gd}$ DTPA was found to correlate with a reduced volume of distribution and suggests that the complex is confined to the blood compartment. $^{42}$

Another strategy to detect $\mathrm{Fe}^{2+}$ ions uses a molecule including $3 \mathrm{Gd}^{3+}$-containing moieties linked together by a trishydroxamate unit, which is a strong binding agent for $\mathrm{Fe}^{2+}$. This approach relies on a concept similar to that schematized in Fig $4 B$; however, here the $\mathrm{Gd}^{3+}$ complexes can be found in 1 molecular entity. This molecule is very flexible, resulting in a limited relaxivity. On chelation of Fe by the tris-hydroxamate, the whole system becomes highly rigid, thereby restricting flexibility of the entire assembly. This results in about $60 \%$ of the relaxivity increase at $20 \mathrm{MHz}$, without changing the molecular weight of the molecule. ${ }^{43}$

Concerning detection of $\mathrm{Fe}^{3+}$, only 1 system has been described on the basis of DTPA - bis salicylamide. The relaxivity increases on the formation of the $\mathrm{Fe}^{3+}$-bound complex due to the increase in the molecular weight. The main drawback of this system is its $\mathrm{pH}$-dependency. ${ }^{44}$

\section{Cu Sensing}

$\mathrm{Cu}$ is the third most abundant transition metal in the body and in the brain, with average neural $\mathrm{Cu}$ concentrations on the order of $0.1 \mathrm{mmol} / \mathrm{L}$. Like $\mathrm{Fe}, \mathrm{Cu}$ can be found in 2 different oxidation states, +1 (cuprous) and +2 (cupric); the former is more common in the reductive intracellular environment, whereas the latter is dominant in the more oxidative extracellular environment. $\mathrm{Cu}$ is partitioned into tightly bound and labile pools, and free $\mathrm{Cu}$ can be released into the synapse on presynaptic excitation, reaching $\leq 15-30 \mu \mathrm{mol} / \mathrm{L}$ in the synaptic cleft. ${ }^{11}$ The normal brain extracellular concentrations are in the range of $0.2-1.7 \mu \mathrm{mol} / \mathrm{L}$, but like $\mathrm{Zn}, \mathrm{Cu}$ was found in high concentrations $(\sim 400 \mu \mathrm{mol} / \mathrm{L})$ in amyloid plaques that are involved in Alzheimer disease. ${ }^{32}$ Disruption of $\mathrm{Cu}$ homeostasis is implicated in other neurodegenerative diseases, including Parkinson disease, familial amyotrophic lateral sclerosis, Menkes disease, and Wilson disease. ${ }^{45}$

No MR imaging contrast agent has been proposed for $\mathrm{Cu}^{+}$ sensing. For $\mathrm{Cu}^{2+}$ detection, a single MR imaging contrast agent has been described in the literature. This $\mathrm{Gd}^{3+}$ complex also functions on the basis of a change in the hydration number on $\mathrm{Cu}^{2+}$ coordination (Fig 4A). The ligand is composed of a macrocyclic core of complex $\mathrm{Gd}^{3+}$ coupled with a pendant iminodiacetate arm for $\mathrm{Cu}^{2+}$ binding. In the presence of $\mathrm{Cu}^{2+}$, this arm de-coordinates from $\mathrm{Gd}^{3+}$, leading to a higher hydration number and a subsequent increase in relaxivity. ${ }^{46}$ In a typical experiment, the addition of $30-\mu \mathrm{mol} / \mathrm{L} \mathrm{Cu}^{2+}$, the estimated concentration released during neural activity, to a $0.21-\mathrm{mmol} / \mathrm{L}$ solution of complex gave a $9 \%$ relaxivity increase, a value which might be suitable for in vivo imaging applications. However, so far, to our knowledge, no in vivo MR imaging experiments have been reported for $\mathrm{Cu}$ sensing.

\section{pH-Sensitive Probes}

The potential use of $\mathrm{pH}$-sensitive probes is vast, though so far $\mathrm{pH}$ mapping of tissues has been mainly intended to facilitate cancer detection and assess the tumor status. Indeed, the $\mathrm{pH}$ 


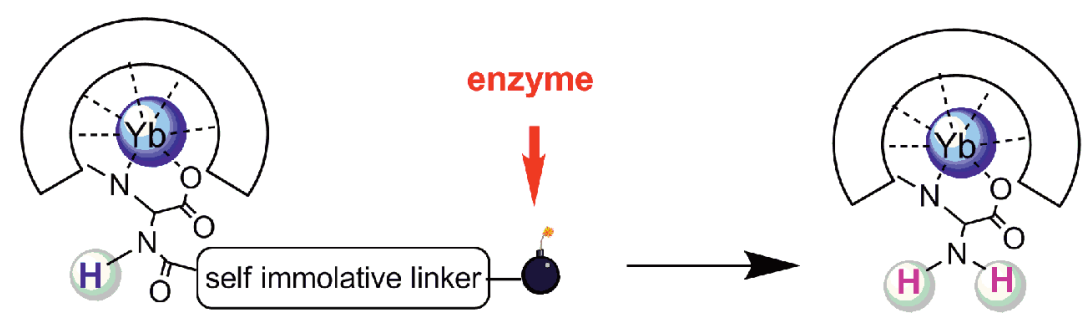

Fig 5. An example of an enzyme-activated PARACEST contrast agent: The enzyme specifically reacts with the substrate leading to the spontaneous elimination of the self-immolative linker This gives rise to a change in the PARACEST properties.

on the surface of tumors is $\sim 0.4$ units lower than that in normal tissue, but in some cases, it can be as low as $6.0 .{ }^{47}$ On the other hand, $\mathrm{pH}$-sensitive probes could also indicate neuronal activity because they induce a slight acidification of the extracellular medium ( $\mathrm{pH} 7.2-7.4) .{ }^{48}$ One could also imagine the use of $\mathrm{pH}$-responsive probes to determine if the brain environment is suitable for a drug that functions in a $\mathrm{pH}$-sensitive environment.

The design of $\mathrm{pH}$-sensing probes has been an intensive field in MR imaging contrast agent research. Nonexhaustive examples are provided. ${ }^{49-55}$ Some of the $\mathrm{pH}$ probes also proved useful for in vivo application. The combination of the $\mathrm{pH}$-sensitive amido-phosphonate derivative of Gd-DOTA and a $\mathrm{pH}$-insensitive analog was used in a dual injection to image renal $\mathrm{pH}$ in mice. ${ }^{56,57}$ The assumption was made that both compounds have comparable pharmacokinetics; hence, the concentration of the former can be inferred from the concentration of the latter. This mixture has been also used to obtain extracellular $\mathrm{pH} \mathrm{MR}$ imaging maps in a rat glioma model, with improved spatial resolution compared with spectroscopic methods. ${ }^{58}$ Differences of the order of $1 \mathrm{pH}$ unit could be detected; the absolute $\mathrm{pH}$ values have been calculated by using a calibration method.

A key factor determining the effectiveness of an agent is the difference between the relaxivity of the "on" state compared with that of the "off" state. Recently, the amplitude of the relaxivity response to $\mathrm{pH}$ variation of this low-molecularweight probe has been largely improved (more than doubled) by conjugating it to a macromolecular dendrimeric scaffold. ${ }^{59}$ However, improving the relaxivity response to $\mathrm{pH}$ through increased molecular weight may also negatively impact the effectiveness of such agents. Large molecules, such as dendrimers, remain in the vasculature longer than discrete agents, which are better able to diffuse into all extracellular space. Furthermore, large molecules also tend to clear more slowly from the body as a result of increased liver uptake. This extends the retention time of $\mathrm{Gd}^{3+}$ in the body. Further studies into the in vivo behavior of dendrimer-based MR imaging contrast media will be required to establish whether this approach, which is successful for increasing the relaxivity response, will yield agents that can actually be applied in vivo.

PARACEST agents have a great potential to indicate $\mathrm{pH}$. The PARACEST effect originates from proton exchange between the paramagnetic probe and water, and the magnitude of the observed effect is intrinsically dependent on the exchange rate, itself related to $\mathrm{pH} .{ }^{60}$ Unlike traditional $\mathrm{Gd}^{3+}$ based agents, it is possible to administer and independently visualize 2 PARACEST agents in the same experiment and thus develop a concentration-independent ratiometric method for $\mathrm{pH}$ determination. For instance, one can acquire the PARACEST pH profiles for a cocktail of 2 isostructural probes, including an identical ligand but 2 different paramagnetic metal ions. The individual PARACEST response of the 2 probes to $\mathrm{pH}$ will be different, whereas their distribution in tissue should be identical. Consequently, the ratio of the 2 PARACEST profiles is concentration-independent and can be used to determine $\mathrm{pH}$ directly without knowing the analytic concentration of either agent. In some cases, the 2 proton pools giving rise to the 2 PARACEST effects can be found within a single molecule, which further simplifies the $\mathrm{pH}$ measurement. ${ }^{4,61,62}$

\section{Enzyme-Activated Contrast Agents}

Among all responsive agents, enzyme targeting represents a specific advantage, which is particularly valid for MR imaging detection, given its low sensitivity. A small concentration of the enzyme can catalytically convert a relatively high amount of the enzyme-responsive magnetic probe, which markedly decreases the limit of detection for the enzyme compared with other biomolecules. In addition, the high specificity of enzymatic reactions means that the observed change in the MR imaging properties can be unambiguously attributed to the targeted enzyme. The presence of certain enzymatic reactions indicates the cellular state and can provide the signature of a given pathology. Therefore, the real-time noninvasive in vivo detection of specific enzymatic activities would have invaluable diagnostic impact. As an example, the importance of enzymatic activity has been established in the processes of tumor formation, growth, and metastasis. Molecular biology studies have defined enzymatic steps of the apoptotic response to anticancer therapies in vitro and in vivo. The detection of gene markers (such as $\beta$-galactosidase) could be another important field of application.

The first enzymatically responsive potential MR imaging contrast agent was a Gd-DOTA - derivative bearing a galactopyranose residue that prevents water coordination. ${ }^{63,64}$ This sugar moiety is a substrate for the enzyme $\beta$-galactosidase. Its enzymatic cleavage by $\beta$-galactosidase opens the access of water to the first coordination sphere of $\mathrm{Gd}^{3+}$, resulting in a relaxivity increase, thus irreversibly activating the agent. It has been successfully used for the in vivo detection of $\beta$-galactosidase messenger ribonucleic acid expression in living Xenopus laevis embryos.

Among other examples, Anelli et $\mathrm{al}^{65}$ functionalized Gd(DTPA $)^{2-}$ with sulfonamide, which is known as a specific carbonic anhydrase inhibitor. The agent reacts with carbonic an- 
hydrase, and thus targets enzyme-rich tissues. Nivorozhkin et $\mathrm{al}^{66}$ reported the enzymatic transformation of a prodrug $\mathrm{Gd}^{3+}$ complex with poor affinity to HSA and low relaxivity to a species with improved HSA affinity and enhanced relaxivity. In this approach, the origin of the relaxivity increase was the slower rotation for protein-bound chelates with respect to the small-molecular-weight prodrug. The same concept was applied for the detection of alkaline phosphatase. ${ }^{67}$ Mazooz et $\mathrm{al}^{68}$ described a Gd-DTPA peptide acting as a transglutaminase substrate, which was used to monitor transglutaminase activity. Shiftan et al reported MR imaging visualization of hyaluronidase in ovarian carcinoma, ${ }^{69}$ related to the aggressiveness of ovarian cancer metastasis. Chen et $\mathrm{a}^{70}$ visualized plaque rupture in atherosclerosis with a Gd-DOTAserotonin derivative, which polymerizes in the presence of neutrophil myeloperoxidase, resulting in a remarkable relaxivity increase. Tyramido or tryptamido derivatives of GdDTPA were proposed as enzyme-activated agents for peroxidase imaging. ${ }^{71}$

Another concept for enzyme detection is based on the selfimmolative mechanism. Duimstra et $\mathrm{al}^{72}$ reported a $\mathrm{Gd}^{3+}$ complex with a self-immolative moiety, designed for the detection of $\beta$-glucuronidase. PARACEST agents with a response to enzymatic activity have also been reported. Yoo and $\mathrm{Pagel}^{73}$ and Yoo et $\mathrm{al}^{74}$ used a thulium-DOTA-monoamide complex containing a peptide chain, which is hydrolyzed by the caspase- 3 enzyme. Following enzymatic cleavage, the PARACEST effect originating from the amide proton disappears due to the hydrolysis of the amide bond.

In another example, the lanthanide complex has been linked to an enzyme-specific substrate via a self-immolative spacer (Fig 5) ${ }^{75}$ With benzyloxycarbamates as a self-immolative unit, the substrate can be any enzyme-recognized moiety capable of transitionally reducing the electron donor capabilities of the phenyl substituent. The enzymatic cleavage of the substrate initiates an electron cascade and leads to the spontaneous elimination of the spacer. This results in the appearance of a PARACEST effect attributed to the exchange of the amine protons. This platform represents several positive features: the substrate is at the extremity of a spacer, which facilitates the enzymatic cleavage, and the PARACEST properties will not be affected by the variation of the substrate. Because the PARACEST effect is observed after enzymatic activation, it can be optimized once for the whole family. The same lanthanide chelate and spacer can be applied for the detection of diverse enzymes, by varying the substrate. The system works as a switch off-on probe, which can be a further advantage in practical in vivo or in vitro applications.

\section{Redox Responsive Agents}

It is widely believed that hypoxia is an important factor governing tumor aggressiveness and that hypoxic tissue is more resistant to conventional therapeutics. ${ }^{76-78}$ Methods for imaging tumor redox status would allow the noninvasive application of this potential biomarker of tumor sensitivity to existing and novel chemotherapies, as well as radiation therapy. The utility of such methods could also extend to other pathologies, such as cardiovascular disease, because free radical formation is associated with deleterious effects on the coronary microcirculation during recovery from myocardial infarction. ${ }^{79}$
Hypoxia is mostly detected by imaging techniques including positron-emission tomography and BOLD MR imaging. Reports on redox-sensitive MR imaging contrast agents have been rather scarce. The simplest design of a redox-responsive MR imaging contrast agent could be based on metal complexes whose metal ion can be reduced or oxidized depending on the biologic environment in which the 2 oxidation forms have different relaxation properties. Thus, the 2 redox states influence the proton relaxation of the surrounding protons to a different extent, resulting in different image intensities. In this perspective, the adducts formed between tpps complexes of $\mathrm{Mn}$ (III) and $\mathrm{Mn}$ (II) and poly- $\beta$-cyclodextrin have been reported to have considerably different relaxivities depending on the redox state of the metal, itself determined by the partial oxygen pressure of the solution. ${ }^{80}$ More recently, DOTAbased complexes of Gd bearing a thiol moiety were synthesized, and it was hypothesized that these complexes would form reversible covalent linkages with HSA, which contains a reactive thiol at cysteine-34. This redox-sensitive reversible binding of Gd complexes to plasma albumin could be exploited for imaging the tissue-redox state. ${ }^{81}$

Recently, the first example of a redox-active responsive PARACEST agent has been reported by Ratnakar et al. ${ }^{82}$ The system is based on a DOTA-derivative Eu complex bearing a nitro group, which can be reduced under hypoxic conditions to an amine. The authors demonstrated that there is a clear difference in the PARACEST signal intensity between the oxidized nitro and the reduced amino derivative. They suggest that the PARACEST difference generated by the chemically reducible probe could be exploited to design biologically applicable redox-responsive imaging agents.

\section{Conclusions}

A large number of activatable smart $\mathrm{Gd}^{3+}$-based or PARACEST MR imaging probes with potential utility for molecular neuroimaging applications have been reported in the literature. So far only a very few of them have progressed to in vivo tests. Future work will likely focus on extending applications of these agents to living animals, as well as on exploring new ways of creating molecular MR imaging probes to meet requirements such as higher specificity or lower detection limits. Ultimately these advances may allow spotting disease-causing abnormalities before any symptoms surface. Molecular imaging agents could speed up the advent of personalized medicine, in which therapies are tailored to individual patients and doctors can monitor the impact of treatment on a molecular scale.

\section{References}

1. Merbach AE, Toth E. The Chemistry of Contrast Agents in Medical Magnetic Resonance Imaging. Chichester, UK: John Wiley \& Sons; 2001

2. Caravan P, Ellison JJ, McMurry TJ, et al. Gadolinium(III) chelates as MRI contrast agents: structure, dynamics, and applications. Chem Rev 1999;99: 2293-352

3. Allen MJ, Meade TJ. Magnetic resonance contrast agents for medical and molecular imaging. Met Ions Biol Syst 2004;42:1-38

4. Aime S, Delli Castelli D, Terreno E. Novel pH-reporter MRI contrast agents. Angew Chem Int Ed Engl 2002;41:4334-36

5. Zhang S, Winter $\mathrm{P}$, Wu K, et al. A novel europium(III)-based MRI contrast agent. J Am Chem Soc 2001;123:1517-18

6. Woods M, Woessner DE, Sherry AD. Paramagnetic lanthanide complexes as PARACEST agents for medical imaging. Chem Soc Rev 2006;35:500-11

7. Woessner DE, Zhang S, Merritt ME, et al. Numerical solution of the Bloch 
equations provides insights into the optimum design of PARACEST agents for MRI. Magn Reson Med 2005;53:790-99

8. Sherry AD, Woods M. Chemical exchange saturation transfer contrast agents for magnetic resonance imaging. Annu Rev Biomed Eng 2008;10:391-411

9. Hammoud DA, Hoffman JM, Pomer MG. Molecular neuroimaging: from conventional to emerging techniques. Radiology 2007;245:21-42

10. Jasanoff A. MRI contrast agents for functional molecular imaging of brain activity. Curr Op Neurobiol 2007;17:593-600

11. Que LE, Domaille DW, Chang CJ, Metals in neurobiology: probing their chemistry and biology with molecular imaging. Chem Rev 2008;108:1517-49

12. Logothetis NK. What we can do and what we cannot do with fMRI. Nature 2008;453:869-78

13. Waters J, Larkum M, Sakmann B, et al. Supralinear $\mathrm{Ca}^{2+}$ influx into dendritic tufts of layer $2 / 3$ neocortical pyramidal neurons in vitro and in vivo. J Neurosci 2003;23:8558-67

14. Kerr JND, Greenberg D, Helmchen F. From the cover: imaging input and output of neocortical networks in vivo. Proc Natl Acad Sci U S A 2005;102: 14063-68

15. Ohki K, Chung S, Ch'ng YH, et al. Functional imaging with cellular resolution reveals precise micro-architecture in visual cortex. Nature 2005;433:597-603. Epub 2005 Jan 19

16. Somjen GG. Ions in the Brain: Normal Function, Seizures, and Stroke. Oxford, UK: Oxford University Press; 2004

17. Koller CW. Neuroprotective therapy for Parkinson's disease. Exp Neurol 1997;144:24-28

18. Panov AV, Burke JR, Strittmatter WJ, et al. In vitro effects of polyglutamine tracts on $\mathrm{Ca} 2+$-dependent depolarization of rat and human mitochondria: relevance to Huntington's disease. Arch Biochem Biophys 2003;410:1-6

19. Yan HD, Lim W, Lee KW, et al. Sera from amyotrophic lateral sclerosis patients reduce high-voltage activated $\mathrm{Ca}^{2+}$ currents in mice dorsal root ganglion neurons. Neurosci Lett 1997;235:69-72

20. Jimerson DC, Post RM, Carman JS, et al. CSF calcium: clinical correlates in affective illness and schizophrenia. Biol Psychiatry 1979;14:37-51

21. Yarlagadda A. Role of calcium regulation in pathophysiology model of schizophrenia and possible interventions. Med Hypotheses 2002;58:182-86

22. Gailly P. New aspects of calcium signaling in skeletal muscle cells: implications in Duchenne muscular dystrophy. Biochim Biophys Acta 2002;1600: $38-44$

23. Lin YJ, Koretsky AP. Manganese ion enhances T1-weighted MRI during brain activation: an approach to direct imaging of brain function. Magn Reson Med $1997 ; 38: 378-88$

24. Koretsky AP, Silva AC. Manganese-enhanced magnetic resonance imaging (MEMRI). NMR Biomed 2004;17:527-31

25. Atanasijevic T, Shusteff M, Fam P, et al. Calcium-sensitive MRI contrast agents based on superparamagnetic iron oxide nanoparticles and calmodulin. Proc Natl Acad Sci U S A 2006;103:14707-712

26. Shapiro MG, Atanasijevic T, Faas $\mathrm{H}$, et al. Dynamic imaging with MRI contrast agents: quantitative considerations. Magn Res Imaging 2006;24:449-62

27. Li W, Fraser SE, Meade TJ. A calcium-sensitive magnetic resonance imaging contrast agent. J Am Chem Soc 1999;12:1413-14

28. Dhingra K, Fousková P, Angelovski G, et al. Towards extracellular Ca2+ sensing by MRI: synthesis and calcium-dependent $1 \mathrm{H}$ and $17 \mathrm{O}$ relaxation studies of two novel bismacrocyclic $\mathbf{G d}^{3+}$ complexes. J Biol Inorg Chem 2008;13:3546. Epub 2007 Sep 15

29. Mishra A, Fousková P, Angelovski G, et al. Facile synthesis and relaxation properties of novel bispolyazamacrocyclic $\mathrm{Gd}(3+)$ complexes: an attempt towards calcium-sensitive MRI contrast agents. Inorg Chem 2008;47:1370-81. Epub 2008 Mar 28

30. Angelovski G, Fousková P, Mamedov I, et al. Smart MRI agents sensing extracellular calcium fluctuations. Chembiochem 2008;9:1729-34

31. Dhingra K, Maier ME, Beyerlein M, et al. Synthesis and characterization of a smart contrast agent sensitive to calcium. Chem Commun (Camb) 2008;344446. Epub 2008 Jun 4

32. Faller P, Hureau C. Bioinorganic chemistry of copper and zinc ions coordinated to amyloid-beta peptide. Dalton Trans 2009:1080-94. Epub 2008 Nov 26

33. Hanaoka K, Kikuchi K, Urano Y, et al. Selective sensing of zinc ions with a novel magnetic resonance imaging contrast agent. J Chem Soc Perkin Trans 2 2001:1840

34. Hanaoka K, Kikuchi K, Urano Y, et al. Design and synthesis of a novel magnetic resonance imaging contrast agent for selective sensing of zinc ion. Chem Biol 2002;9:1027-32

35. Major JL, Boiteau RM, Meade TJ. Mechanisms of ZnII-activated resonance imaging agents. Inorg Chem 2008;47:10788 -95. Epub 2008 Oct 18

36. Major JL, Parigi G, Luchinat C, et al. The synthesis and in vitro testing of a zinc-activated MRI contrast agent. Proc Natl Acad Sci U S A 2007;104:13881-86

37. Trokowski R, Ren J, Kalman FK, et al. Selective sensing of zinc ions with a PARACEST contrast agent. Angew Chem Int Ed Engl 2005;44:6920-23

38. Paris J, Gameiro C, Humblet V, et al. Auto-assembling of ditopic macrocyclic lanthanide chelates with transition-metal ions: rigid multimetallic high relaxivity contrast agents for magnetic resonance imaging. Inorg Chem 2006;45: 5092-102
39. Livramento JB, Toth E, Sour A, et al. High relaxivity confined to a small molecular space: a metallostar-based, potential MRI contrast agent. Angew Chem Int Ed Engl 2005;44:1480 - 84

40. Livramento JB, Sour A, Borel A, et al. A starburst-shaped heterometallic compound incorporating six densely packed $\mathrm{Gd}(3+)$ ions. Chemistry 2006;12: 989-1003

41. Livramento JB, Weidensteiner C, Prata MI, et al. First in vivo MRI assessment of a self-assembled metallostar compound endowed with a remarkable high field relaxivity. Contrast Media Mol Imaging 2006;1:30-39

42. Parac-Vogt TN, Vander Elst L, Kimpe K, et al. Pharmacokinetic and in vivo evaluation of a self-assembled gadolinium(III)-iron(II) contrast agent with high relaxivity. Contrast Med Mol Imaging 2006;1:267-78

43. Jacques V, Desreux JF. New classes of MRI contrast agents. Top Curr Chem 2002;221:125-64

44. Aime S, Botta M, Fasano M, et al. Paramagnetic GdIII-FeIII heterobimetallic complexes of DTPA-bis-salicylamide. Spectrochimica Acta 1993;9:1315-22

45. Gaggelli E, Kozlowsky H, Valensin D, et al. Copper homeostasis and neurodegenerative disorders (Alzheimer's prion, and Parkinson's diseases and amyotrophic lateral sclerosis). Chem Rev 2006;106:1995-2044

46. Que EL, Chang CJ. A smart magnetic resonance contrast agent for selective copper sensing. J Am Chem Soc 2006;128:15942-43

47. Gillies RJ, Raghunand N, Karczmar GS, et al. MRI of the tumor microenvironment. J Magn Reson Imaging 2002;16:430-50

48. Chesler M. Regulation and modulation of $\mathrm{pH}$ in the brain. Physiol Rev 2003;83:1183-221

49. Kalman FK, Woods M, Caravan $\mathrm{P}$, et al. Potentiometric and relaxometric properties of a gadolinium-based MRI contrast agent for sensing tissue $\mathrm{pH}$. Inorg Chem 2007;46:5260-70

50. Aime S, Crich SG, Gianolio E, et al. High sensitivity lanthanide(III) based probes for MR-medical imaging. Coord Chem Rev 2006;250:1562-79

51. Mikawa M, Miwa N, Brautigam M, et al. A pH-sensitive contrast agent for functional magnetic resonance imaging (MRI). Chem Lett 1998;7:693-94

52. Aime S, Crich SG, Botta M, et al. A macromolecular Gd(III) complex as pHresponsive relaxometric probe for MRI applications. Chem Commun 1999:1577-78

53. Lowe MP, Parker D, Reany O, et al. pH-dependent modulation of relaxivity and luminescence in macrocyclic gadolinium and europium complexes based on reversible intramolecular sulfonamide ligation. J Am Chem Soc 2001;123: 7601-09

54. Hovland R, Glogard C, Aasen AJ, et al. Gadolinium DO3A derivatives mimicking phospholipids; preparation and in vitro evaluation as $\mathrm{pH}$ responsive MRI contrast agents. J Chem Soc Perkin Trans 2 2001;6:929-33

55. Toth E, Bolskar RD, Borel A, et al. Water-soluble gadofullerenes: toward highrelaxivity, pH-responsive MRI contrast agents. J Am Chem Soc 2005;127:799805

56. Zhang S, Wu K, Sherry AD. A novel pH-sensitive MRI contrast agent. Angew Chem Int Ed Engl 1999;38:3192-94

57. Raghunand N, Howison C, Sherry AD, et al. Renal and systemic $\mathrm{pH}$ imaging by contrast-enhanced MRI. Magn Reson Med 2003;49:249-57

58. Garcia-Martin ML, Martinez GV, Raghunand N, et al. High-resolution pH(e) imaging of rat glioma using $\mathrm{pH}-$ dependent relaxivity. Magn Reson Med 2006;55:309-15

59. Ali MM, Woods M, Caravan P, et al. Synthesis and relaxometric studies of a dendrimer-based pH-responsive MRI contrast agent. Chemistry 2008;14: $7250-58$

60. Ward KM, Balaban RS. Determination of $\mathrm{pH}$ using water protons and chemical exchange dependent saturation transfer (CEST). Magn Reson Med 2000;44:799-802

61. Aime S, Barge A, Delli Castelli D, et al. Paramagnetic lanthanide(III) complexes as $\mathrm{pH}$-sensitive chemical exchange saturation transfer (CEST) contrast agents for MRI applications. Magn Reson Med 2002;47:639-48

62. Terreno E, Delli Castelli D, Cravotto G, et al. Ln(III)-DOTAMGly complexes: a versatile series to assess the determinants of the efficacy of paramagnetic chemical exchange saturation transfer agents for magnetic resonance imaging applications Invest Radiol 2004;39:235-43

63. Moats RA, Fraser SE, Meade TJ. A “smart" magnetic resonance imaging agen that reports on specific enzymatic activity. Angew Chem Int Ed Engl 1997;36: $726-28$

64. Louie AY, Hüber MM, Ahresn ET, et al. In vivo visualization of gene expression using magnetic resonance imaging. Nat Biotechnol 2000;18:321-25

65. Anelli PL, Bertini I, Fragai M, et al. Sulfonamide-functionalized gadolinium DTPA complexes as possible contrast agents for MRI: a relaxometric investigation. Eur J Inorg Chem 2000:625-30

66. Nivorozhkin AL, Kolodziej AF, Caravan P, et al. Enzyme-activated Gd(III) magnetic resonance imaging contrast agents with a prominent receptor-induced magnetization enhancement. Angew Chem Int Ed Engl 2001;40:2903-06

67. Lauffer RB, McMurry TJ, Dunham SO, et al. Epix Medical Inc. Bioactivated diagnostic imaging contrast agents. Patent publication number WO9736619. October 9, 1997

68. Mazooz G, Mehlman T, Lai TS, et al. Development of magnetic resonance 
imaging contrast material for in vivo mapping of tissue transglutaminase activity. Cancer Res 2005;65:1369-75

69. Shiftan L, Israely T, Cohen M, et al. Magnetic resonance imaging visualization of hyaluronidase in ovarian carcinoma. Cancer Res 2005;65:10316-23

70. Chen JW, Pham W, Weissleder R, et al. Human myeloperoxidase: a potential target for molecular MR imaging in atherosclerosis. Magn. Reson Med 2004;52:1021-28

71. Querol M, Chen JW, Weissleder R, et al. DTPA-bisamide-based MR sensor agents for peroxidase imaging. Org Lett 2005;7:1719-22

72. Duimstra JA, Femia FJ, Meade TJ. A gadolinium chelate for detection of betaglucuronidase: a self-immolative approach. J Am Chem Soc 2005;127:12847-55

73. Yoo B, Pagel MD. A PARACEST MRI contrast agent to detect enzyme activity. J Am Chem Soc 2006;128:14032-33

74. Yoo B, Raam MS, Rosenblum RM, et al. Enzyme-responsive PARACEST MRI contrast agents: new biomedical imaging approach for studies of the proteasome. Contrast Media Mol Imaging 2007;2:189-98

75. Chauvin T, Durand P, Bernier M, et al. Detection of enzymatic activity by PARACEST MRI: a general approach to target a large variety of enzymes. Angew Chem Int Ed Engl 2008;47:4370-72
76. Raghunand N, Gatenby RA, Gillies RJ. Microenvironmental and cellular consequences of altered blood flow in tumours. Br J Radiol 2003;76:S11-S22

77. Rofstad EK. Microenvironment-induced cancer metastasis. Int J Radiat Biol 2000;76:589-605

78. Raghunand N, Gillies RJ. pH and drug resistance in tumors. Drug Resist Updat 2000;3:39-47

79. Matsumoto $\mathrm{H}$, Inoue $\mathrm{N}$, Takaoka $\mathrm{H}$, et al. Depletion of antioxidants is associated with no-reflow phenomenon in acute myocardial infarction. Clin Cardiol 2004;27:466-70

80. Aime S, Botta M, Gianolio E, et al. A pO2-responsive MRI contrast agent based on the redox switch of manganese (II/III)-porphyrin complexes. Angew Chem Int Ed 2000;39:747-50

81. Raghunand N, Jagadish B, Trouard TP, et al. Redox-sensitive contrast agents for MRI based on reversible binding of thiols to serum albumin. Magn Reson Med 2006;55:1272-80

82. Ratnakar SJ, Woods M, Lubag AJ, et al. Modulation of water exchange in europium(III) DOTA-tetraamide complexes via electronic substituent effects. J Am Chem Soc 2008;130:6-7. Epub 2007 Dec 8 